\title{
EVOLUÇÃO DO CONHECIMENTO SOBRE PROJETOS COMPLEXOS: UMA ANÁLISE DA PRODUÇÃo CIENTÍFICA
}

\section{KNOWLEDGE EVOLUTION ON COMPLEX PROJECTS: A SCIENTIFIC PRODUCTION ANALYSIS}

\section{Frederico Steffen Neto}

Discente do Mestrado Profissional em Administração - Gestão de Projetos (MPA-GP/UNINOVE), São Paulo. Professor da Faculdades Integradas Torricelli, Guarulhos.

E-mail: fredericosteffen@gmail.com (Brasil)

\section{Marcos Roberto Piscopo}

Doutorado em Administração pela Universidade de São Paulo - USP, São Paulo.

Docente do Programa de Pós-graduação em Administração (PPGA/UNINOVE) e Mestrado Profissional em Administração - Gestão de Projetos (MPA-GP/UNINOVE), São Paulo.

E-mail: piscopomr@gmail.com (Brasil) 


\section{EVOLUÇÃO DO CONHECIMENTO SOBRE PROJETOS COMPLEXOS: UMA ANÁLISE DA PRODUÇÃO CIENTÍFICA}

\section{RESUMO}

A literatura define projeto complexo como aquele que atende a determinadas e específicas características. Outro fator que pode levar um projeto a ser considerado complexo é a forma eficaz de gerenciar os riscos existentes - atividade esta que requer do gestor do projeto ações que vão além daquelas analíticas e simples. Este trabalho objetiva estudar a evolução do conhecimento científico sobre projetos complexos no período de 2003 a 2013. Para a realização deste trabalho foi realizado um estudo através de documentação secundária, em que se definiu o período a ser estudado, selecionado as bases de dados a serem exploradas, identificados os periódicos com relevância para o tema projetos complexos, e identificados os artigos por meio do uso de palavras-chave. Os resultados sugerem que o tema projetos complexos ainda é incipiente, com publicações efetuadas predominantemente nos últimos cinco anos, em periódicos internacionais, concentradas em um pequeno grupo de autores que geraram a maior parte das citações.

Palavras-chave: Projetos Complexos, Produção Científica, Conhecimento Científico, Gestão de projetos. 


\title{
KNOWLEDGE EVOLUTION ON COMPLEX PROJECTS: A SCIENTIFIC PRODUCTION ANALYSIS
}

\begin{abstract}
The literature defines complex project as one that meets certain and specific characteristics. Another factor that may lead a project to be considered complex is the effective way to manage risks, wich requires the project manager to go beyond those analytical and simple actions. This work aims to study the evolution of scientific knowledge on complex projects from 2003 to 2013. To achieve this goal it was conducted a documental study by defining the time frame to be researched, the databases to be explored, the relevant journals to the complex projects theme and the articles to be investigated according to the keywords. Results suggest that complex projects theme is still incipient, with publications distributed predominantly in the last five years, in international journals, and concentrated in a small group of authors who generated most of the citations.
\end{abstract}

Keywords: Complex Projects, Scientific Production, Scientific Knowledge, Project Management. 


\section{INTRODUÇÃO}

Historicamente considera-se que as origens do paradigma da complexidade remontam às pesquisas realizadas no Biological Computer Laboratory. Em 1956, Heinz Von Foerster em parceria com renomados cientistas - aprofundou temas como causalidade circular, auto referência e o papel organizador do acaso, mixando conhecimento da biologia e da cibernética (Serva, 1992). Complexidade não possui uma definição única e simples, quando aplicada ao gerenciamento de projetos, referindo-se neste caso ao comportamento e desempenho de equipes virtuais, não reflete necessariamente projetos complicados e grandes, nem implica dificuldade técnica. A teoria da complexidade ou teoria do caos é uma teoria relativamente nova, tendo suas raízes na matemática e nas ciências exatas; moveu-se de forma lenta para as ciências sociais e empresas, e encontra-se na busca de seu lugar na gestão de projetos (Rose, 2011). Dado o fato de que projetos se encontram inseridos no ambiente organizacional em que estão ocorrendo, por usar equipamentos e conter pessoas em seu contexto, podemos relacionálos com a teoria do caos, eis que as organizações possuem comportamentos muito mais complexos se comparadas com as máquinas, pois as organizações são compostas por seres humanos. Um dos principais desafios das organizações é acompanhar a evolução de seus ambientes. São duas as principais dificuldades para ultrapassar esses desafios: chegar a algum estado estável, acreditando isso ser possível através da adaptação às mudanças ocorridas no ambiente externo; e por acreditarem as organizações que ações e decisões conduzam aos resultados esperados (Naveira, 1998).

Projetos podem ser tipificados como complexos em decorrência de algumas características ou dimensões. Um projeto pode ser considerado complexo desde que ele atenda uma ou mais das seguintes características: tecnológica, social, financeira, legal, organizacional e temporal (Hertogh \& Westerveld, 2010). Ainda, a complexidade de projetos pode ser definida como o composto de muitas partes inter-relacionadas, diversas e que podem ser operacionalizadas em termos de diferenciação e interdependência (Baccarini, 1996).

Nas últimas décadas muitos projetos foram realizados ao redor de todo o mundo, muitas discussões a respeito da complexidade desses projetos foram levantadas, nos mais diversos segmentos de negócios. Diversos estudiosos, das mais diferentes linhas de estudo e mais variadas formações acadêmicas, analisando a complexidade desses projetos, dedicaram seu tempo a estudar e produzir uma gama de material sobre projetos complexos. Exemplos desses trabalhos incluem estudos de gerenciamento de projeto, não se limitando porém a isso 
propriamente (Baccarini, 1996), mas abrangem estudos sobre os fatores de risco (Thamhain, 2013), gerenciamento dos stakeholders e gerenciamento de tempo (Baccarini, 1996), e também gerenciamento de custos e sustentabilidade (Davies \& Hobday, 2005). Dessa forma, estudar a complexidade de projetos tem sido muito importante, não só para a academia como também para as empresas e gestores de projetos, pois o conhecimento sobre os fatores de complexidade pode auxiliar na gestão de novos projetos, permitindo mitigar riscos oriundos da complexidade.

Em decorrência da importância relevante dos projetos complexos, em âmbito nacional e internacional, surge a necessidade de se analisar o conhecimento sobre a gestão desses projetos com o propósito de melhor conhecer a literatura existente e entender os principais desafios dessa área de pesquisa. Por conta disso, este trabalho visa avaliar a evolução do conhecimento científico sobre projetos complexos, no período de 2003 a 2013. Mais especificamente, procurou-se analisar o número de artigos publicados por periódico, se tais periódicos possuem fator de impacto ou indexação, a origem dos periódicos consultados e dos artigos publicados, o ano de publicação dos artigos, os autores dos artigos, os métodos de estudos utilizados e o número de citações dos artigos.

O presente trabalho está estruturado em cinco etapas. Na primeira etapa, contextualizouse o tema e apresentou-se o objetivo do estudo. A etapa 2 aborda a revisão teórica sobre projetos complexos. A terceira etapa discorre sobre os procedimentos metodológicos empregados. A quarta etapa contempla a apresentação e a análise dos resultados. Finalmente, na $5^{\text {a }}$. etapa, são colocadas as conclusões do estudo, bem como suas contribuições, limitações e sugestões de ampliação.

\section{REFERENCIAL TEÓRICO}

O suporte teórico deste artigo aborda os principais aspectos conceituais de projetos complexos e gestão desses empreendimentos.

\subsection{COMPLEXIDADE NO AMBIENTE DE NEGÓCIOS}

A teoria da complexidade englobou em seu bojo a teoria do caos, fractais, teoria das catástrofes, lógicas/conjuntos difusos dentre outras, procedentes das ciências exatas que se direcionam, de forma implícita e explícita, cada vez mais para uma visão mais realista, sem 
simplificação, sem reducionismo; de forma paradoxal essas teorias nos últimos anos vêm se aproximando das ciências humanas e naturais. Essas ciências estão sendo utilizadas para melhor entender as estruturas e os processos organizacionais multímodo, ultrapassando as teorias clássicas sobre organizações (Tôrres, 2005).

De acordo com a teoria do caos, sistemas de grande interesse e tão diferentes, desiguais, como a economia ou o cérebro humano, são desordenados em sua essência. A partir do momento em que se esclarecem os mecanismos por trás desses comportamentos, ela elucida a compreensão de suas dinâmicas. A teoria traz à luz novas perspectivas para a modelagem de sistemas não lineares, que constituem regra no mundo real. Muito embora essa luz seja uma nova forma de olhar a realidade, ela não pode ser desprezada, resta um enorme caminho a ser trilhado para se obterem as respostas certas (Wood Jr., 1993).

Sistemas adaptativos multifacetados conseguem fracionar uma situação intrincada em vários elementos, que apesar de nunca se combinarem daquela forma, já foram percebidos antes. Assim como nesses sistemas, os projetos apesar de serem naturalmente únicos por si só, e não terem sido executados antes nas mesmas condições, também possuem vários desses elementos definidos para rearranjo e aplicação em novas situações (Sato, Dergint, \& Hatakeyama, 2004).

\subsection{PROJETOS COMPLEXOS}

Projetos têm sido realizados desde há muito tempo, sendo que alguns dentre eles, em decorrência de suas características, são vistos e tratados como labirínticos. A literatura nos possibilita identificar quando possuem esse talante ou não, de acordo com uma série de peculiaridades previstas e analisadas, bem como avaliar a questão da heterogeneidade na gestão deles. Baccarini (1996) define projetos quanto à complexidade quando são compostos por diversas partes inter-relacionadas e pode ser operacionalizado em termos de diferenciação e interdependência.

Tais peculiaridades caracterizam projetos como sistemas compostos por diversas e interligadas partes, com a capacidade de mudar e aprender com a experiência; assim tem havido um interesse crescente em ideias relacionadas à teoria da complexidade, como forma de compreensão e raciocínio sobre as características e dinâmica dos projetos, e, ainda, aqueles que apresentam atributos específicos de raridade, restrição, multidisciplinaridade, complexidade tecnológica, administrativa e/ou objetivos dos stakeholders (Hass, 2009). 
De acordo com Lima e Farias (2012), citados por (Hertogh \& Westerveld, 2010), projetos complexos se valem da combinação de abordagem baseada na vivência, na qual se destacam seis dimensões. São elas: tecnológica, social, financeira, legal, organizacional e temporal. A complexidade dos projetos pode ainda ser apreciada por seu alto valor e alta tecnologia, definidos como de alto custo, de engenharia, software, bens e sistemas de uso intensivo. Podem estar inclusos nesta classe vários tipos de bem de capital, redes, sistemas, subsistemas e construções de engenharia (Davies \& Hobday, 2005). Essa conceituação requer uma capacidade de gerenciar as reivindicações contraditórias dos stakeholders e o uso do mapeamento de argumentos pode auxiliar nesse problema, oferecendo uma mutável cartografia móvel ou de colaboração científica, estruturando o conhecimento das justificativas e refutações inerentes ao que foi dito pelos interessados (Metcalfe \& Sastrowardoyo, 2013).

De acordo com o modelo Diamante (Shenhar \& Dvir, 2007), a multiplicidade de um projeto pode ser caracterizada por quatro dimensões: I) complexidade estrutural (baixa: montagem; média: sistema e alta: ordem); II) novidade ou inovação (derivado-plataformadescoberta); III) ritmo (regular-rápido-repentino); e IV) tecnologia (baixo-médio-altosuperalto). De acordo com os autores, a partir dessas quatro variáveis é possível gerar métricas para classificar o grau de dificuldade do projeto, proporcionando orientações para discussão de risco nesse cenário.

\subsection{GESTÃO DE PROJETOS COMPLEXOS}

Administrar uma organização, e consequentemente projetos, é uma atividade que a cada dia se defronta com novos desafios, os quais podem ter origem a partir da aceleração contínua de mudanças, da disponibilidade de grande volume de dados, da presença massiva dos meios de comunicação e da quebra das fronteiras comerciais. Por conta disso, pode-se afirmar que os ambientes de negócios e dos projetos estão se tornando obscuros.

Para estudar e entender esses espaços surge a teoria da complexidade ou simplesmente complexidade, composta por um conjunto de conceitos que procuram esclarecer os fenômenos naturais em sua totalidade. Trata-se de uma ciência descritiva e explicativa (Giovannini, 2002).

O gerenciamento de projetos ganhou importância ao longo das últimas décadas e é cada vez mais comum em muitos tipos de organizações. Atualmente, há uma preocupação com a relevância das abordagens mais convencionais para esse gerenciamento, para problemas que 
são cada vez mais complexos e limitadores, e que envolvem um grande número de partes interessadas ou stakeholders (Davis, MacDonald, \& White, 2010).

Complementarmente aos projetos em estudo também tem sido identificada a importância da gestão daqueles, para a qual alguns fatores são evidentes: sua complexidade ajuda a determinar os requisitos de controle, de planejamento e coordenação, dificulta a identificação clara de metas e objetivos de grandes projetos, é um critério importante na seleção de uma forma apropriada de organização, influencia na seleção dos inputs (requisitos de competência e experiência de gestão de pessoal), é frequentemente usada como critério para a seleção de fornecedores adequados, e, finalmente, a complexidade afeta as variáveis de tempo, custo e qualidade. Geralmente quanto maior a complexidade maior será o tempo e o custo do projeto (Baccarini, 1996).

As pesquisas referentes à gestão são algo relativamente recentes, normalmente caracterizadas por meio de métodos de reestruturação da administração e adequação de técnicas especiais que pretendem possibilitar a obtenção de melhor controle e utilização dos recursos existentes. $\mathrm{O}$ gerenciamento objetiva uso dos recursos existentes valendo-se do fluir dos trabalhos nos sentidos horizontal e vertical dentro da organização; de forma geral o fluxo vertical é realizado pelos gerentes de linha e o horizontal pelos gerentes de projetos sendo seu esforço principal a comunicação e coordenação das atividades horizontalmente entre as organizações de linha (Kerzner, 2011).

Projetos de alta diversidade pressupõem a integração baseada no conhecimento de equipes; com igualdade de participação, podendo ser introduzida a noção de equipe segregada para explicar esse conhecimento (Enberg, Lindkvist \& Tell, 2010). É importante para o gerenciamento identificar o mais cedo possível os sinais de alerta. Os métodos atuais de avaliação que possibilitam distinguir esses sinais são estabelecidos como uma ferramenta para os proprietários para que possam ter certeza de que as decisões tomadas são fundamentadas em suficiente número de fatos e análises, as quais geralmente são realizadas como parte dos procedimentos e ancoradas em estruturas de governança estabelecidas (Williams, Klakegg, Walker, Andersen, \& Magnussen, 2012).

Ainda em relação a esse tema, a literatura discute determinados tipos de modelos para o acompanhamento tradicional dos projetos: orçamento, cronograma e escopo. Apesar de ser reconhecida a importância da integração na gestão, foco deste estudo, pouco tem sido escrito a respeito. A administração moderna de projetos exige de seus praticantes habilidades de 
integração, onde se sabe que o gerente de projeto global enfrenta problemas de integração especialmente difíceis (Cioffi, 2002).

Conforme Zhai, Xin, e Chaosheng (2009), essa atividade pode gerar valor significativo para as organizações, sua valorização varia de acordo com os diferentes tamanhos e a complexidade dos projetos gerenciados. Outro fator importante para o sucesso ou falha de projetos é a liderança exercida sobre eles em todos os níveis da empresa. A qualidade da liderança na administração e controle é fator-chave na implementação de uma estratégia empresarial (Cleland \& Ireland, 1999).

Deve-se levar em consideração outro aspecto nos projetos complexos, que é saber lidar de forma eficaz com seus riscos, atividade difícil e que vai exigir por parte do gestor intervenções que vão além das abordagens analíticas simples. A gestão eficaz e eficiente dos riscos envolve um conjunto de variáveis ligadas de forma intrínseca, relacionadas ao processo, às pessoas, e ao ambiente organizacional. Alguns projetos de sucesso apontaram para a importância de reconhecer e gerenciar os riscos no início de seu desenvolvimento (Thamhain, 2013).

Tem-se notado que com a ascendência da complexidade dos projetos e processos de negócios os gestores estão mais conscientes do enredamento das variáveis de riscos entre os sistemas e processos organizacionais, o que limita a eficiência dos métodos analíticos. Muitos gestores ponderam, por vezes, que inexistem nas organizações pessoas ou grupo de pessoas que conheçam e possuam conhecimento de causa para avaliar esses riscos de múltiplas variáveis e seus efeitos em cascata (Thamhain, 2013).

Segundo Yugue e Maximiano (2013), de forma geral, a dimensão metas de desempenho é uma das mais importantes em relação à contribuição para a complexidade do projeto. Quando da definição do escopo observa-se seu nível de diversidade, uma vez que é nesse processo que essas características são consideradas. Estas não estão relacionadas a algumas dimensões específicas, mas a um conjunto de dimensões; aparentemente também é plausível considerar nessa avaliação aspectos pessoais como parte da percepção da complexidade de tal projeto.

Nos dias atuais, diferentemente do que ocorria no passado, os gerentes de projetos se valem de computadores e tecnologias avançados para lidar de forma eficaz com os riscos que podem ser identificados e descritos de forma analítica, utilizando métodos estatísticos, simulações de cenários de negócios e desenho centrado no usuário (Thamhain, 2013). Ainda, segundo o autor, diversas organizações têm desenvolvido seus próprios procedimentos, 
normas, políticas e ferramentas de gestão para lidar com os riscos, tendo como foco suas próprias necessidades e situações.

Conforme Remington e Pollack (2007), mesmo nos dias atuais a gestão de projetos é praticada e ensinada com base em teorias que foram desenvolvidas no início do período moderno, nos séculos XIX e XX, para se relacionar com a expansão imperial e a industrialização, independentemente do porte e da complexidade; e com relação à gestão em ambientes complexos, é necessária a capacidade de observar os sistemas a partir de diferentes perspectivas e aplicar ferramentas e metodologias adequadas à situação específica.

O moderno gerenciamento, de forma contínua revisitada e aperfeiçoada, segundo Valeriano (2005), pode ser instrumento capaz de materializar os produtos do conhecimento de forma eficiente e rápida. Tempos atrás, o hiato existente entre os avanços científicos e tecnológicos e sua aplicação era longo, fato que hoje praticamente inexiste, a aproximação dessa linha de atuação com as frentes de desenvolvimentos diminuiu essa lacuna.

Algum tempo atrás se argumentava que a maioria das definições na literatura sobre competências em gerenciamento de projetos eram baseadas em características funcionais, formadas por um conjunto de quatro elementos e suas relações: tecnologia, pessoas, estrutura e cultura organizacional, possibilitando a discussão sobre diferentes tipos de competências que passam desde as competências mais simples até aquelas mais complexas. É comum supor que competências essenciais são impossíveis de se imitar. Ao assumir esse tipo de pensamento é natural concluir que seu desenvolvimento, e eventual substituição, não é um problema para a investigação e/ou de gestão de atenção (Drejer, 2001).

A academia nos apresenta algumas situações com relação ao tópico: a definição de complexo não resiste a uma investigação, não houve nenhuma análise dos problemas que o estabelecimento desta iniciativa destina-se a resolver, o processo pelo qual a academia e a norma têm progredido foi desmarcado, e o padrão utilizado não é estabelecido com práticas baseadas em evidências (Whitty \& Maylor, 2009).

Apesar de o Guia Pmbok apresentar inúmeras informações e orientações para os gerentes, existe a oportunidade de incluir questões pertinentes à teoria da complexidade. No ambiente externo (Guia Pmbok®, 2008) é possível aos gestores encontrarem uma riqueza de recursos e diferenciadas formas de organizações de gerenciamento ao redor do mundo. Um gerente tem de determinar se está interessado em material para organizações virtuais ou não virtuais e deve ir à busca de uma organização adequada (Curlee \& Gordon, 2010). 


\section{METODOLOGIA}

A bibliometria surgiu no início do século devido à necessidade de se avaliar e estudar as produções científicas. Entende-se como sendo uma técnica quantitativa e estatística de medição dos índices de produção e disseminação do conhecimento científico (Araújo, 2006). É um conjunto de leis e princípios empíricos que colaboram e contribuem para o estabelecimento do fundamento teórico da ciência da informação (Guedes \& Borschiver, 2005). O termo mais utilizado para defini-la é aquele elaborado por Pritchard (1969), como todos os estudos que tentam quantificar os processos de comunicação escrita, é amplo e objetivo, pois de forma determinada esclarece a essência dos estudos bibliométricos.

Para a elaboração de trabalhos que procuram quantificar os produtos da atividade científica, é importantíssimo fazer uso dos preceitos de Lotka (1926), que se refere ao cálculo da produtividade de autores de artigos científicos; Bradford (1953), sobre a dispersão dos autores em diferentes publicações periódicas; Zipf (1949) quanto à frequência de ocorrência de palavras em um texto longo; e Price (1963) que, a partir dos trabalhos de Lotka, Bradford e Zipf, formulou as leis cienciométricas centradas fundamentalmente na análise da dinâmica da atividade científica, tanto os produtos quanto os produtores da ciência. (Santos \& Kobashi, 2009).

A operacionalização deste trabalho envolveu um conjunto de etapas. Primeiramente, definiu-se o intervalo entre janeiro/2003 e dezembro/2013 como período a ser avaliado. Em seguida foram definidas as bases de dados Web of Science, Spell e Google Scholar como fontes de pesquisa para identificação e seleção dos artigos a serem examinados, considerando a relevância para o tema projetos complexos e a disponibilidade eletrônica para condução da pesquisa. O uso dessas justifica-se pelo fato de que a Web of Science é reconhecida mundialmente, com publicações dos principais journals internacionais; a Spell é a mais importante no que se refere a dados nacionais, disponibilizando publicações dos journals mais relevantes e a Google Scholar pelo fato de ser um site de livre acesso com grande abrangência.

A busca dos artigos foi direcionada com a utilização dos seguintes termos: projetos complexos, complex projects, complexidade de projetos, project complexity, complexity of projects, complexidade do produto do projeto, project product complexity, complexidade na gestão de projetos e project management complexity. Em seguida, do rol de artigos selecionados, foram eliminadas as duplicidades, e aqueles publicados nos periódicos com 
qualificação Qualis inferior a B3 na área de administração, ciências contábeis e turismo. Finalmente realizou-se doublecheck da qualificação dos artigos consolidando as informações obtidas do Qualis-Capes e do Web of Science.

Encerrada a fase de qualificação e seleção final dos papers que cumpriram com os critérios definidos, identificou-se em quais Journals ocorreram essas publicações, os quais estão relacionados na Figura 1. Para os 71 artigos efetivamente selecionados analisaram-se: I) a quantidade de artigos publicados por periódico; II) se os periódicos consultados possuem fator de impacto ou indexação; III) a origem dos periódicos (internacional ou nacional); IV) a origem dos artigos publicados nos periódicos (internacional ou nacional); v) o ano de publicação dos artigos, dentro do período definido; VI) os métodos de estudo empregados nos artigos; VII) o número de citações recebidas pelos artigos; e VIII) a rede de cocitações entre os autores. Para a geração do item de análise (VIII) foi feito uso dos softwares BibExcel e Ucinet, gerando inicialmente um arquivo bibliográfico a partir do site Web of Science. Em uma segunda etapa o arquivo gerado sofreu tratamento no aplicativo BibExcel e deu origem a uma matriz de relacionamento entre os autores indicados nas referências bibliográficas. Na terceira e última etapa, as ligações identificadas foram inseridas no aplicativo Ucinet para assim produzir uma representação gráfica dos vínculos existentes entre as referências bibliográficas indicadas em cada um dos artigos utilizados.

\section{Figura 1 - Relação dos periódicos pesquisados}

\begin{tabular}{|c|c|}
\hline African Journal of Business Management & Journal of the Operational Research Society \\
\hline Decision Sciences & Project Management Journal \\
\hline $\begin{array}{l}\text { Economic Computation and Economic Cybernetics Studies } \\
\text { and Research }\end{array}$ & Public Management Review \\
\hline European Journal of Operational Research & $R \& D$ Management \\
\hline Group Decision and Negotiation & R.Adm. \\
\hline Iberoamerican Journal of Project Management & RAE. Revista de Administração de Empresas \\
\hline Ieee Transactions on Engineering Management & RAM. Revista de Administração Mackenzie \\
\hline Industrial Marketing Management & Regional Science and Urban Economics \\
\hline Information Systems Research & Revista de Administração e Inovação \\
\hline Interfaces & Revista de Gestão da Tecnologia e Sistemas de Informação \\
\hline $\begin{array}{lllll}\text { International Journal of Operations \& Production } \\
\text { Management }\end{array}$ & Revista de Gestão e Projetos - GeP \\
\hline International Journal of Project Management & Revista Gestão \& Tecnologia \\
\hline International Journal of Technology Management & Revista Ibero-Americana de Estratégia - RIAE \\
\hline Journal of Business Economics and Management & Revista Produção Online \\
\hline Journal of Business Research & Revue D'Études Comparatives Est-Ouest \\
\hline Journal of Economic Interaction and Coordination & $\begin{array}{l}\text { South African Journal of Economic and Management } \\
\text { Sciences }\end{array}$ \\
\hline Journal of Engineering and Technology Management & System Dynamics Review \\
\hline Journal of International Management & Systemic Practice and Action Research \\
\hline
\end{tabular}




\begin{tabular}{|l|l||}
\hline Journal of Management Information Systems & Technological and Economic Development of Economy \\
\hline Journal of Operations Management & Technology Analysis \& Strategic Management \\
\hline
\end{tabular}

Fonte: Elaborado pelos autores.

\section{APRESENTAÇÃO E ANÁLISE DOS RESULTADOS}

Após análise dos 71 artigos selecionados, os dados foram estudados e os resultados são apresentados a seguir em conformidade com os seguintes tópicos: artigos por periódico, fator de impacto ou qualificação webqualis, origem dos journals, origem dos artigos, ano de publicação dos artigos, autores dos artigos, método de estudo dos artigos e cocitações dos artigos.

\subsection{ARTIGOS POR PERIÓDICO}

$\mathrm{Na}$ Tabela 1 apresentamos a quantidade de artigos por periódico com o intuito de analisar a existência ou não de concentração de estudos em específicos agentes publicadores.

Tabela 1 - Artigos por agente publicador

\begin{tabular}{||l|c|c|}
\hline \hline \multicolumn{1}{|c|}{ Publicações } & Quantidade & \% de participação \\
\hline International Journal of Project Management & 10 & $14 \%$ \\
\hline Project Management Journal & 9 & $13 \%$ \\
\hline Journal of the Operational Research Society & 5 & $7 \%$ \\
\hline European Journal of Operational Research & 3 & $4 \%$ \\
\hline African Journal of Business Management & 2 & $3 \%$ \\
\hline Ieee Transactions on Engineering Management & 2 & $3 \%$ \\
\hline Revista de Gestão e Projetos - GeP & 2 & $3 \%$ \\
\hline Journal of Management Information Systems & 2 & $3 \%$ \\
\hline R \& D Management & 2 & $3 \%$ \\
\hline Revista Produção Online & 2 & $3 \%$ \\
\hline Demais Publicações (com 1 artigo) & 32 & $45 \%$ \\
\hline Total geral & 71 & $100 \%$ \\
\hline \hline
\end{tabular}

Fonte: Elaborado pelos autores.

A análise da quantidade de artigos publicados por periódico demonstra elevada concentração, visto que dos 71 trabalhos selecionados, 39 foram publicados em apenas 10 deles, conforme demonstrado na Tabela 1 . Assim, nota-se que $55 \%$ do total dos estudos foram publicados em $24 \%$ do número de journals. Adicionalmente, ressalta-se que apenas dois destes publicaram 19 artigos, correspondendo a $27 \%$ do número total. São eles o International 
Journal of Project Management e o Project Management Journal, considerados veículos científicos especializados no campo da gestão de projetos.

Quanto aos 32 periódicos restantes, cada um publicou um artigo no período considerado, totalizando 32 estudos. Esses dados estão consistentes com a lei de Bradford, a qual possibilita estimar o grau de relevância de periódicos em determinadas áreas de conhecimento, onde os que produzem o maior número de artigos sobre dado assunto formam um núcleo de estudos (Guedes \& Borschiver, 2005), demonstrando que ainda existe certa concentração, mas em contrapartida estão surgindo novos interessados em divulgar estudos concernentes ao assunto projetos complexos, ou gestão de projetos complexos.

\subsection{FATOR DE IMPACTO E $Q U A L I S$-CAPES}

Por meio da Tabela 2 identificamos quantos artigos, daqueles utilizados, possuem fator de impacto ou classificação Qualis-Capes.

\section{Tabela 2 - Fator de impacto e WEBQUALIS-Capes}

\begin{tabular}{|c|c|c|}
\hline \hline Classificação & Quantidade & \% de participação \\
\hline NA & 19 & $45 \%$ \\
\hline A1 & 6 & $14 \%$ \\
\hline A2 & 4 & $10 \%$ \\
\hline B1 & 3 & $5 \%$ \\
\hline B2 & 2 & $7 \%$ \\
\hline B3 & 3 & $12 \%$ \\
\hline Fator de impacto & 5 & $100 \%$ \\
\hline Total geral & 42 & \\
\hline \hline
\end{tabular}

Nota: NA = Periódicos que não possuem fator de impacto e não possuem extrato Qualis.

Fonte: Elaborado pelos autores.

Sobre esse quesito, conforme apontado na Tabela 2, observa-se que 55\% dos periódicos avaliados possuem fator de impacto e ou qualificação Qualis, segundo consulta realizada ao Journal Citation Reports da Thomson Reuters e ao WebQualis-Capes.

O campo da gestão de projetos tem recebido crescente atenção da academia, contudo ainda se nota o predomínio dos veículos mais direcionados aos praticantes. Especificamente no que se refere ao tema projetos complexos, observa-se que quantidade razoável das publicações tem ocorrido em journals sem fator de impacto, pois sua natureza mais 
tecnológica e menos científica facilita o acesso dos praticantes. Entretanto pode-se observar que $26 \%$ dos artigos foram publicados naqueles de classificação A1 (seis artigos) ou com fator de impacto (cinco artigos), denotando procura pelos autores de agentes publicadores com alto reconhecimento. Apesar do foco (técnico, tecnológico ou praticante), o tema tem recebido atenção crescente da Academia em razão da busca por periódicos de alta qualificação.

\subsection{ORIGEM DOS PERIÓDICOS}

Na Tabela 3 apresentamos a segregação da origem dos periódicos, para analisar a quantidade de estudos divulgados por agentes publicadores nacionais e internacionais.

Tabela 3 - Origem do periódico

\begin{tabular}{|c|c|c|}
\hline \hline Origem & Quantidade & \% de participação \\
\hline Nacional & 9 & $21 \%$ \\
\hline Internacional & 33 & $79 \%$ \\
\hline Total geral & 42 & $100 \%$ \\
\hline \hline
\end{tabular}

Fonte: Elaborado pelos autores.

Verifica-se que 33 dos 42 periódicos selecionados e avaliados neste estudo são de origem internacional. O campo da gestão de projetos emergiu com o programa aeroespacial americano, a partir do qual se desenvolveram muitos estudos. Complementarmente, ressaltase que os journals International Journal of Project Management e o Project Management Journal são internacionais e publicaram $27 \%$ do número total de trabalhos avaliados na presente pesquisa. Destaca-se que, no Brasil, o campo do gerenciamento se encontra em desenvolvimento e que o número de agentes publicadores cujo escopo envolve o referido tema ainda é reduzido. Assim, trabalhos sobre esse tema são direcionados para aqueles da área de gestão tecnológica. Percebe-se a real possibilidade para preenchimento da lacuna existente no tocante à quantidade de publicadores de estudos referentes a projetos complexos e, também, sobre sua gestão. 


\subsection{ORIGEM DOS ARTIGOS}

Da mesma forma que na Tabela 3, na Tabela 4 segregou-se em duas linhas de classificação, nacional e internacional, porém nesta o foco de identificação e classificação foram os próprios artigos.

Tabela 4 - Origem dos artigos

\begin{tabular}{|c|c|c|}
\hline \hline Origem & Quantidade & \% de participação \\
\hline Nacional & 11 & $15 \%$ \\
\hline Internacional & 60 & $85 \%$ \\
\hline Total geral & 71 & $100 \%$ \\
\hline
\end{tabular}

Fonte: Elaborado pelos autores.

Com respeito à origem dos artigos, nota-se a partir da Tabela 4, que esses são predominantemente internacionais, aspecto que reforça o entendimento apresentado no tópico 4.3. que analisou a origem dos periódicos. Dessa forma, os autores dos artigos pesquisados buscam referências internacionais sobre o tema projetos complexos e também direcionam seus trabalhos para journals internacionais, os quais apresentam maior oportunidade de acomodação desses esforços, assim como o reconhecimento dos autores fora de suas próprias fronteiras.

\subsection{ANO DE PUBLICAÇÃO DOS ARTIGOS}

A partir da Tabela 5 objetivou-se apresentar a quantidade de artigos por ano de publicação, fazendo uso do período estudado, de 2003 a 2013.

Tabela 5 - Ano de Publicação dos Artigos

\begin{tabular}{|c|c|c|}
\hline \hline Ano de publicação & Quantidade & \% de participação \\
\hline 2003 & 5 & $7 \%$ \\
\hline 2004 & 4 & $6 \%$ \\
\hline 2005 & 3 & $4 \%$ \\
\hline 2006 & 3 & $4 \%$ \\
\hline 2007 & 4 & $6 \%$ \\
\hline 2008 & 9 & $13 \%$ \\
\hline 2009 & 8 & $11 \%$ \\
\hline 2010 & 10 & $14 \%$ \\
\hline
\end{tabular}




\begin{tabular}{|c|c|c|}
\hline 2011 & 5 & $7 \%$ \\
\hline 2012 & 10 & $14 \%$ \\
\hline 2013 & 10 & $14 \%$ \\
\hline Total geral & 71 & $100 \%$ \\
\hline
\end{tabular}

Fonte: Elaborado pelos autores.

Observa-se que o tema projetos complexos tem sido explorado recentemente. Aponta-se que somente nos últimos três anos foram publicados em torno de $35 \%$ dos artigos avaliados nesse estudo. Se considerado o período de cinco anos, o referido percentual eleva-se para $61 \%$. O crescimento do interesse recente dos pesquisadores e praticantes pelo tema pode estar associado à busca pelo aprofundamento do conhecimento na área que tem como consequência o desenvolvimento de práticas aplicáveis à realidade organizacional. Apesar desse recente interesse por parte dos pesquisadores pondera-se, também, sobre a existência de um espectro considerável de tópicos dentro das áreas de conhecimento da gestão de projetos para estudos daqueles complexos.

\subsection{AUTORES DOS ARTIGOS}

Neste tópico, por meio da Tabela 6, apresentamos a quantidade de artigos por autor.

\section{Tabela 6 - Autores dos Artigos}

\begin{tabular}{|c|c|c|}
\hline \hline Autor & Quantidade & \% de participação \\
\hline Terry Williams & 4 & $2,6 \%$ \\
\hline S. Howick & 3 & $1,9 \%$ \\
\hline C. Eden & 2 & $1,3 \%$ \\
\hline Harvey Maylor & 2 & $1,3 \%$ \\
\hline Kenneth H. Rose & 2 & $1,3 \%$ \\
\hline Roberto Sbragia & 2 & $91,6 \%$ \\
\hline Demais autores com apenas um artigo & 139 & $100,0 \%$ \\
\hline Total geral & 154 & \\
\hline
\end{tabular}

Fonte: Elaborado pelos autores.

Os dados apresentados na Tabela 6 demonstram que existe concentração entre um pequeno grupo de oito autores, em que a quantidade de artigos publicados varia de dois a quatro artigos durante o período considerado para a análise. A maior parte dos trabalhos foi distribuída entre um grande número de autores com autoria de um único artigo. O resultado 
encontrado está de acordo com a lei de Lotka, que preconiza que alguns pesquisadores, de renome em determinadas áreas do conhecimento, produzem o maior número de estudos sobre um determinado assunto, ou seja, pesquisadores supostamente de maior prestígio em uma determinada área do conhecimento produzem muito e muitos pesquisadores, considerados talvez de menor prestígio, produzem pouco, (Guedes \& Borschiver, 2005). Complementarmente observa-se que não é incomum os trabalhos serem realizados em parcerias, com participação de dois ou mais autores. Para o total de 71 artigos constata-se a participação de 154 autores, sendo a média de mais do que dois autores por trabalho.

\subsection{TÓPICOS DE INTERESSE}

Na Tabela 7 apresentamos a quantidade de artigos agrupados por tópico de interesse de estudo.

Tabela 7 - Grupos de Tópicos de Estudos

\begin{tabular}{|c|c|c|}
\hline Grupos de tópicos de estudo & Participação & $\%$ \\
\hline Metodologias de gerenciamento de projetos & 31 & $44 \%$ \\
\hline Gestão estratégica de projetos & 15 & $21 \%$ \\
\hline Equipes de projetos & 5 & $7 \%$ \\
\hline TI aplicada a projetos & 4 & $6 \%$ \\
\hline Ciclo de vida do projeto & 3 & $4 \%$ \\
\hline Gestão do conhecimento em projetos & 3 & $4 \%$ \\
\hline Riscos & 3 & $4 \%$ \\
\hline Gestão de prazo & 2 & $3 \%$ \\
\hline Gerente de projetos & 2 & $3 \%$ \\
\hline Ambiente de negócios & 2 & $3 \%$ \\
\hline Comunicações & 1 & $1 \%$ \\
\hline Total geral & 71 & $100 \%$ \\
\hline
\end{tabular}

Fonte: Elaborado pelos autores.

Em relação ao espectro dos tópicos estudados pelos autores, conforme mencionado no item 4.5, após análise dos 71 artigos selecionados para este trabalho foi possível agrupar em 11 grupos de assuntos, o que permitiu observar que dois desses grupos foram os mais estudados no período pesquisado (gestão estratégica de projetos e metodologias de gerenciamento de projetos) totalizando 46 estudos, ou seja, 65\% do total dos artigos considerados neste trabalho. Tal constatação demonstra que a preocupação maior não é mais em relação às conservadoras tríplices variáveis: cronograma, custo e equipe. 


\subsection{MÉTODO DE ESTUDO}

Da mesma forma que é importante sabermos quantos artigos foram publicados no período, por autor, por ano de publicação, se possuem ou não fator de impacto/indexação, quais os tópicos relacionados a projetos complexos foram estudados, saber qual o método de pesquisa adotado pelos estudiosos também auxilia a compreender como a pesquisa tem sido realizada.

\section{Tabela 8 - Métodos de estudos}

\begin{tabular}{|c|c|c|}
\hline Método de estudo & Participação & $\%$ \\
\hline Estudo de caso múltiplo & 24 & $34 \%$ \\
\hline Estudo de caso único & 23 & $32 \%$ \\
\hline Estudo teórico & 19 & $27 \%$ \\
\hline Survey & 4 & $6 \%$ \\
\hline Método misto & 1 & $1 \%$ \\
\hline Total geral & 71 & $100 \%$ \\
\hline
\end{tabular}

Fonte: Elaborado pelos autores.

Observa-se a aplicação dos métodos mais usuais, em que o estudo de caso único e estudo de casos múltiplos predominam, sendo que o somatório do percentual de participação dessas duas formas de estudo representa mais do que a metade do total dos artigos analisados e estudados, ou seja, 66\%. Como é uma área ainda em expansão e em busca de consolidação os trabalhos são predominantemente de natureza exploratória e descritiva com abordagem qualitativa por meio de estudos de casos.

\subsection{CITAÇÕES DOS ARTIGOS}

A quantidade de citações para cada um dos artigos utilizados neste estudo são apresentadas na Tabela 9.

Tabela 9 - Número de citações por artigo

\begin{tabular}{||l|c|c||}
\hline \hline \multicolumn{1}{|c|}{ Título do artigo } & $\begin{array}{c}\text { Número de } \\
\text { citações }\end{array}$ & $\begin{array}{c}\text { \% de } \\
\text { participação }\end{array}$ \\
\hline Leadership competency profiles of successful project managers & 132 & $11 \%$ \\
\hline Team cognition: Development and evolution in software project teams & 102 & $8 \%$ \\
\hline Exploring interorganizational conflict in complex projects & 95 & $8 \%$ \\
\hline
\end{tabular}




\begin{tabular}{|l|c|c|}
\hline And then came Complex Project Management (revised) & 81 & $6 \%$ \\
\hline How do organizations learn lessons from projects - And do they? & 68 & $5 \%$ \\
\hline $\begin{array}{l}\text { Procuring complex performance in construction: London Heathrow Terminal 5 } \\
\text { and a Private Finance Initiative hospital }\end{array}$ & 47 & $4 \%$ \\
\hline $\begin{array}{l}\text { The effects of information technology project complexity on group interaction } \\
\text { failure in complex projects: Some implications for organizational research }\end{array}$ & 41 \\
\hline $\begin{array}{l}\text { Designing teams for speedy product development: The moderating effect of } \\
\text { technological complexity }\end{array}$ & 40 \\
\hline $\begin{array}{l}\text { Learning from projects } \\
\text { Design and Analysis of Contracts for Software Outsourcing }\end{array}$ & $3 \%$ \\
\hline $\begin{array}{l}\text { Team flexibility's relationship to staffing and performance in complex projects: An } \\
\text { empirical analysis }\end{array}$ & 30 \\
\hline Demais Artigos & 36 & $3 \%$ \\
\hline Total geral & 488 & $3 \%$ \\
\hline \hline
\end{tabular}

Fonte: Elaborado pelos autores.

Com referência às citações resultantes dos artigos publicados, na Tabela 9, observa-se que doze artigos correspondem a $61 \%$ do número total, sendo que apenas três deles representam $26,1 \%$ do volume total considerado. Tais resultados sugerem que o tema ainda é pouco explorado e que um reduzido número de trabalhos é utilizado por grande parte dos autores que publicam sobre projetos complexos.

A Tabela 10 apresenta a quantidade de artigos para uma mesma faixa de número de citações.

Tabela 10 - Quantidade de artigos por números de citações

\begin{tabular}{|c|c|c|}
\hline \hline $\mathbf{N}^{\mathbf{0}}$ de citações & Quantidade & \% de participação \\
\hline de 0 a 4 & 35 & $49 \%$ \\
\hline de 5 a 10 & 5 & $7 \%$ \\
\hline de 11 a 30 & 15 & $21 \%$ \\
\hline de 31 a 50 & 11 & $15 \%$ \\
\hline de 51 a 100 & 3 & $4 \%$ \\
\hline mais que 100 & 2 & $3 \%$ \\
\hline Total geral & 71 & $100 \%$ \\
\hline \hline
\end{tabular}

Fonte: Elaborado pelos autores.

Complementando a Tabela 9, na Tabela 10 observa-se que 56\% dos artigos se encontram entre aqueles não citados ou, com no máximo, 10 citações realizadas. Deixando de forma explícita que ainda é menor a procura de artigos relacionados a projetos complexos. 


\subsection{REDE DE COCITAÇÕES}

Nos 71 artigos validados e utilizados neste trabalho foram identificadas 1.001 referências bibliográficas, onde por meio do uso dos softwares BibExcel e Ucinet foi possível totalizar 42.478 cocitações.

\section{Figura 2 - Rede de Cocitações entre os artigos referenciados duas ou mais vezes}

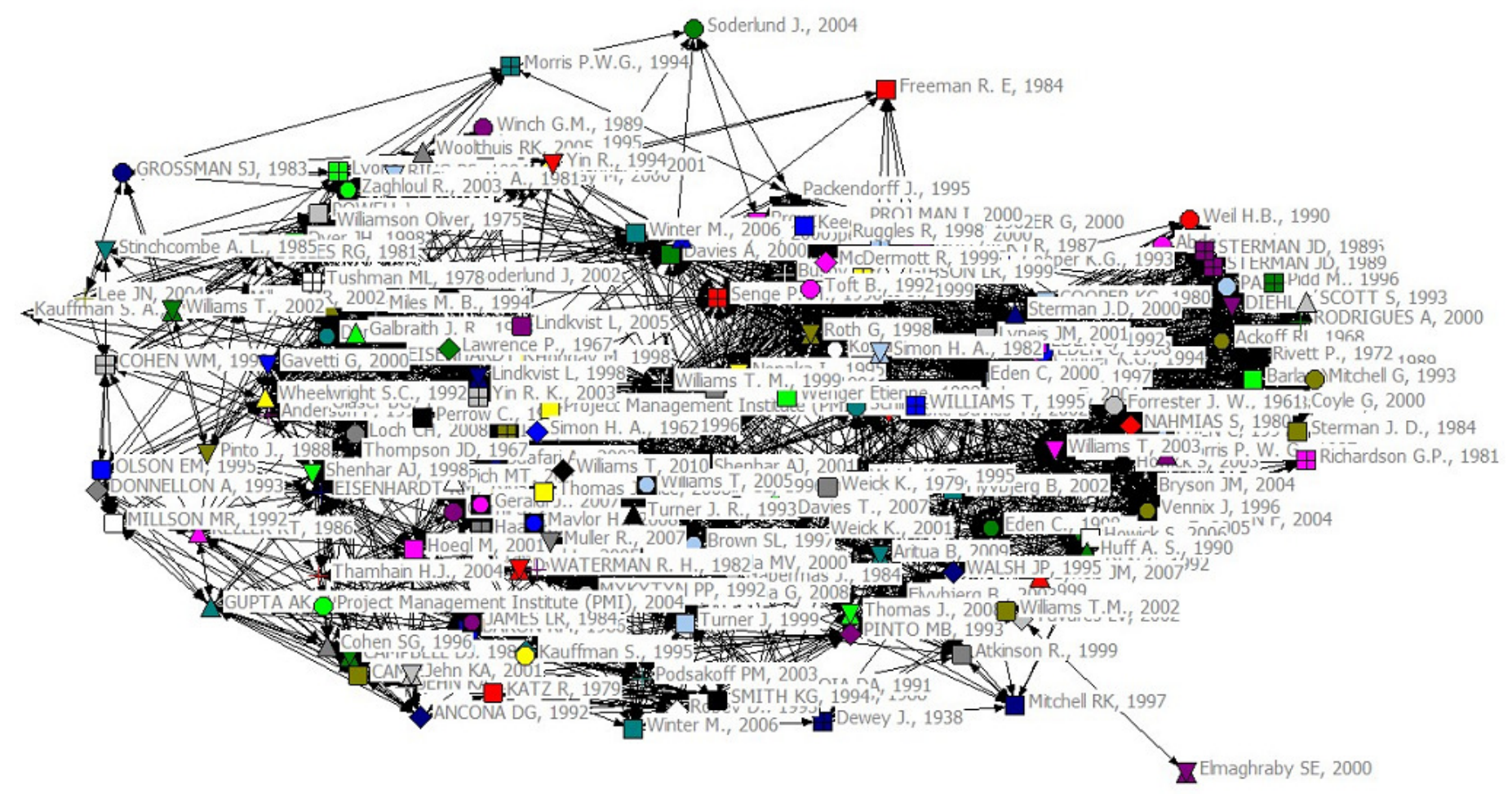

Fonte: Elaborado pelos autores.

Para a geração da Figura 2, realizou-se recorte considerando artigos que foram indicados duas vezes ou mais como referência bibliográfica, totalizando 199 (19,88\%) do número total, que somaram $8.472(19,94 \%)$ cocitações. Dentro dos critérios adotados as mais cocitadas foram: Ackermann F., 1997 (182), Eden C., 2000 (170), Lyneis JM, 2001 (136), Willians T. M., 1999 (120), Cooper K. G., 1994 (118), Forrester J.W., 1961 (110), Eden C., 1992 (103), Williams T. M., 2003 (102).

Por meio da Figura 3, mostra-se o relacionamento existente entre os oito autores mais referenciados nos 71 artigos analisados; observa-se, também, a existência de um círculo de pesquisas entre eles. 


\section{Figura 3 - Rede de Cocitações entre os oito artigos mais cocitados}

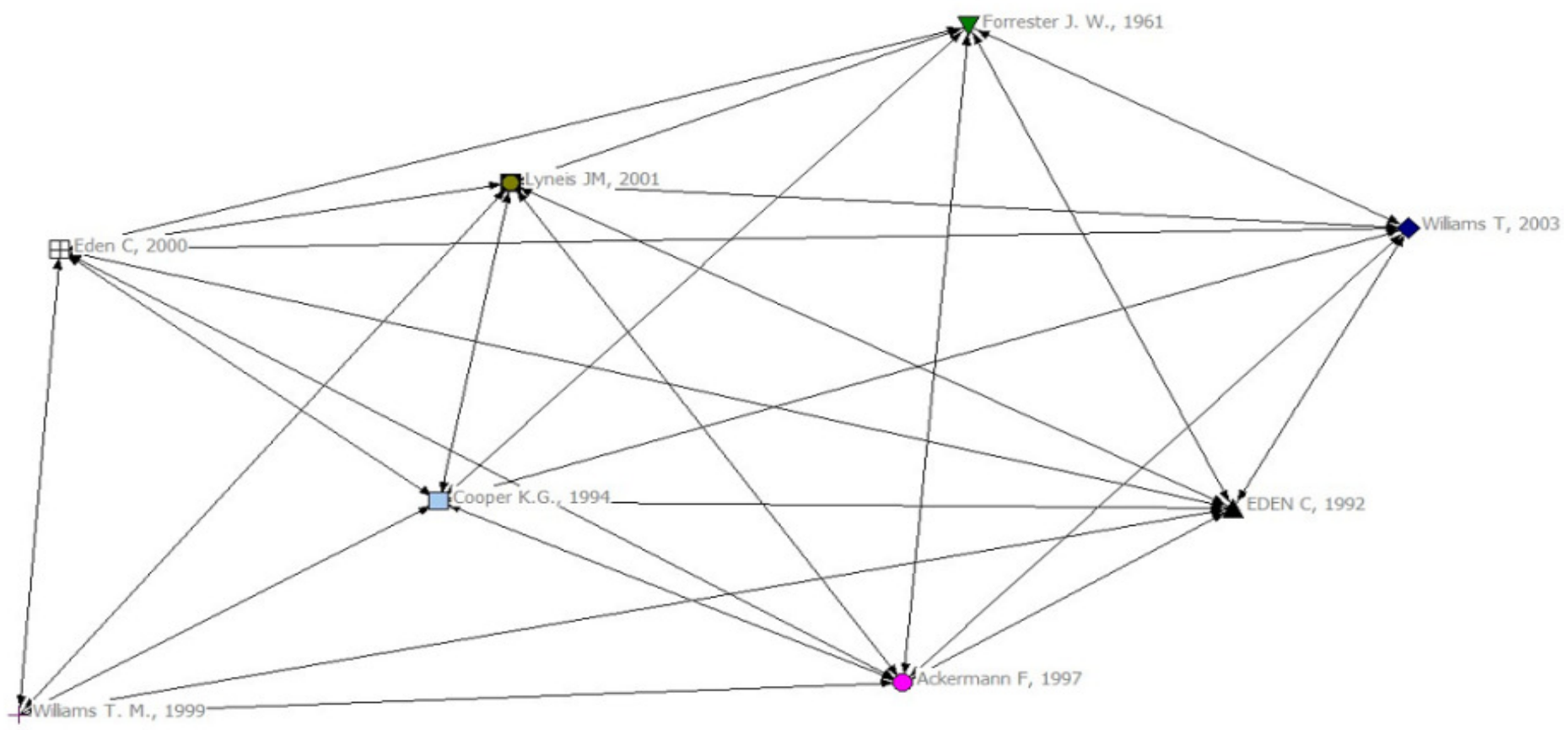

Fonte: Elaborado pelos autores.

Por outro lado, averiguando as Figuras 2 e 3, nota-se que o conhecimento está sendo disseminado junto a outros estudiosos, visto a grande quantidade de cocitações representadas na Figura 2, e a partir desses novos núcleos outros círculos de pesquisas estão se formando, demonstrando resultados consistentes com os apresentados nos tópicos 4.1 e 4.6.

\subsection{ANÁLISE CONSOLIDADA}

Nota-se, de forma visível, a desproporção encontrada entre a produção nacional e a internacional - a internacional dominando plenamente visto que $79 \%$ dos periódicos são internacionais. De maneira decorrente faz com que o mesmo fenômeno se apresente com a origem dos trabalhos publicados, ou seja, predominância de artigos internacionais, representando $85 \%$ das publicações no período.

Outro fato importante que devemos destacar é que a grande maioria dos periódicos, nos quais os estudos foram publicados, não possui fator de impacto ou extrato Qualis, muito embora isso não desmereça sua importância. Isso talvez possa expressar a preocupação dos pesquisadores do tema projetos complexos em apresentar seus estudos em um periódico de alto rigor para aprovação e publicação dos trabalhos. 
Com relação ao período definido para pesquisa e seleção dos papers, de janeiro/2003 a dezembro/2013, houve elevação do número de trabalhos publicados a partir do ano 2008 inclusive, o que demonstra aparentemente aumento no interesse no objeto em questão. Quanto à quantidade de citações se observa que são poucas, sendo que mais da metade dos artigos pesquisados não foram citados, ou o foram somente uma vez. Destaca-se que dois deles foram citados mais que 100 vezes, 35 artigos entre 5 e 100 citações e 27 entre 0 e 4 respectivamente. Vale ressaltar que talvez o número reduzido de trabalhos considerados se deva ao fato dos critérios adotados para este estudo bibliométrico, consultadas somente as bases de dados Web of Science, Spell e Google Scholar, o que limita a sua extensão. Constata-se que a participação de autores ou periódicos nacionais ainda é diminuta frente à participação internacional, fazendo com que se perceba uma lacuna a ser preenchida, possibilitando dessa forma estudos referentes a projetos complexos nas mais diferentes frentes de conhecimento. Destaca-se ainda como ponto importante a constatação dos principais métodos utilizados, quais sejam: estudo de caso único e estudo de caso múltiplo, primordialmente, abordagens qualitativas, provocadas talvez pela dificuldade na obtenção de dados quantitativos sobre projetos complexos.

Os indicadores obtidos neste estudo permitem observarmos que o tema projetos complexos no período pesquisado (2003/2013) foi objeto de poucos trabalhos. Porém em contrapartida, de forma gradativa, nota-se que o interesse por parte da academia vem aumentando principalmente nos últimos cinco anos, com uma pequena contração no ano de 2011, fato que sugere que este tema pode ser alvo de interesse ainda maior por parte dos estudiosos em gestão de projetos nos anos futuros.

\section{CONSIDERAÇÕES FINAIS}

Neste trabalho, foi realizada atividade de levantamento da produção científica relacionada a projetos complexos, no âmbito nacional e internacional. Observa-se que a quantidade de artigos pertinentes ao assunto objeto da pesquisa ainda é incipiente, apesar do interesse demonstrado pela academia.

Como limitações desta pesquisa destacam-se o período de abrangência - 10 anos - e o rigor adotado na seleção dos periódicos e trabalhos conforme metodologia adotada, valendose exclusivamente de consulta nos sites Web of Science, Spell e Google Scholar, e qualificação Qualis superior a B3. 
Este estudo não teve a pretensão de cobrir lacunas que possam existir no estudo referente a projetos complexos, mas o seu pretenso objetivo foi avaliar o quanto se tem discutido a respeito desse assunto no período determinado para pesquisa. Como colaboração para a academia e gestão profissional de projetos, deixamos a sugestão de dar-se continuidade à pesquisa sobre o assunto, abrangendo um período de tempo maior, bem como usar outras bases de dados, de forma tal que possam ser identificados outros estudos, possibilitando assim um aprofundamento no estudo de projetos complexo.

\section{Referências}

Araújo, C. A. Á. (2006). Bibliometria: evolução histórica e questões atuais. Em Questão, 12(1), 11-32.

Baccarini, D. (1996). The concept of project complexity - a review. International Journal of Project Management, 14(4), 201-204.

Bradford, S. C. (1953). Documentation. London: Crosby Lockwood; Washington, Public Affairs Press.

Cioffi, D. F. (2002). Managing project integration. Vienna, VA 22182: Management Concepts.

Cleland, D. \& Ireland, L. (1999). Project management: strategic design and implementation (3nd edition). USA: MacGraw-Hill Companies, Inc.

Curlee, W. \& Gordon, R. L. (2010). Complexity theory and project management. New Jersey: John Wiley \& Sons Inc.

Davies, A. \& Hobday, M. (2005). The business of projects: managing innovation in complex products and systems. The Edinburgh Building, Cambridge: Cambridge University Press.

Davis, J.; MacDonald, A. \& White, L. (2010). Problem-structuring methods and project management: an example of stakeholder involvement using hierarchical process methodology. Journal of the Operational Research Society, 61(6), 893-904.

Drejer, A. (2001). How can we define and understand competencies and their development? Technovation, 21(3), 135-146.

Enberg, C. Lindkvist, L. \& Tell, F. (2010). Knowledge integration at the edge of technology: on teamwork and complexity in new turbine development. International Journal of Project Management, 28(8), 756-765. 
Giovannini, F. (2002). A complexidade e o estudo das organizações: explorando possibilidades. Rausp-Revista de Administração, 37(3), 56-66.

Guedes, V. L. S. \& Borschiver, S. (2005). Bibliometria: Uma ferramenta estatística para a gestão da informação e do conhecimento, em sistemas de informação, de comunicação e de avaliação científica e tecnológica. Anais do VI Cinform Encontro Nacional de Ciência da Informação, Salvador-BA: ICI/UFBA.

Guia Pmbok®, P. (2008). Um guia do conhecimento em gerenciamento de projetos (4a ed.). São Paulo: PMI - Project Management Institute.

Hass, K. B. (2009). Managing complex projects: a new model. Vienna, VA 22182: Managemente Concepts.

Hertogh, M. J. C. M. \& Westerveld, E. (2010). Playing with complexity, management and organisation of large infrastructure projects. RePub, Erasmus University Rotterdam.

Kerzner, H. (2011). Gerenciamento de projetos - uma abordagem sistêmica para planejamento, programação e controle (10a ed.). São Paulo: Editora Edgard Blücher Ltda.

Lima, M. L. A. \& Farias, J. R. de F. (2012). A gestão integrada no gerenciamento de projetos complexos. Anais do VIII Congresso nacional de excelência em gestão, Rio de Janeiro.

Lotka, A. J. (1926). The frequency distribution of scientific productivity. Journal of the Washington Academy of Sciences, vol.16, n.12, pp. 317-323.

Metcalfe, M. \& Sastrowardoyo, S. (2013). Complex project conceptualisation and argument mapping. International Journal of Project Management, 31(8), 1129-1138.

Naveira, R. B. (1998). Caos e complexidade nas organizações. RAP-Revista de Administração Pública, Rio de Janeiro, 32(5), 69-80.

Price, J. D. S. (1963). Litle science, big science. New York, Columbia University Press.

Pritchard, A. (1969). Statistical bibliography or bibliometrics? Journal of Documentation, 25(4), 348-349.

Rabechini Junior, R. \& Carvalho, M. M. de C. (2003). Perfil das competências em equipes de projetos. RAE Eletrônica, 2 (1), 17.

Remington, K. \& Pollack, J. (2007). Tools for complex projects. England: Gower Publishing Limited.

Rose, K. H. (2011). Complexity theory and project management. Project Management Journal, 42(5), 92. 
Santos, R. N. M. dos \& Kobashi, N. Y. (2009). Bibliometria, cientometria, infometria: conceitos e aplicações. Tendências da pesquisa brasileira em ciência da informação, 2 (1), $155-172$.

Sato, C. E. Y.; Dergint, D. E. A. \& Hatakeyama, K. (2004). A organização baseada em projetos (OBP) como um sistema adaptativo complexo (SAC) (p.16). Anais do XXIII Simpósio de gestão da inovação tecnológica, Curitiba - PR.

Serva, M. (1992). O paradigma da complexidade e a análise organizacional. Revista de Administração de Empresas/Eaesp/FVG, 32(2), 26-35.

Shenhar, A. J. \& Dvir, D. (2007). Reinventing project management: the diamond approach to successful growth and innovation. Boston, Massachusetts, USA: Harvard Business School Press.

Thamhain, H. (2013). Managing risks in complex projects. Project Management Journal, 44 (2), 20-35.

Tôrres, J. J. M. (2005). Teoria da complexidade: uma nova visão de mundo para a estratégia. Curitiba - PR: I Ebec - PUC/PR - $1^{\circ}$ Encontro Brasileiro de Estudos da Complexidade.

Valeriano, D. L. (2005). Moderno gerenciamento de projetos (1a ed). Brasil: Prentice Hall Brasil.

Whitty, S. J. \& Maylor, H. (2009). And then came complex project management revised. International Journal of Project Management, 27(3), 304-310.

Williams, T.; Klakegg, O. J.; Walker, D. H. T.; Andersen, B., \& Magnussen, O. M. (2012). Identifying and acting on early warning signs in complex projects. Project Management Journal, 43(2), 37-53.

Wood Jr., T. (1993). Caos: a criação de uma nova ciência? As aplicações e implicações da teoria do caos na administração de empresas. Revista de Administração de Empresas/Eaesp/FGV, 33(4), 94-105.

Yugue, R. T. \& Maximiano, A. C. A. (2013). Entendendo e gerenciando a complexidade de projetos. Revista de Gestão e Projetos - GeP, 4(1), 01-22.

Zhai, L., Xin, Y. \& Chaosheng, C. (2009). Understanding the value of project management from a stakeholder's perspective: case study of mega-project management. Project Management Journal, 40(1), 99-109.

Zipf, G. K. (1949). Human behavior and the principle of least effort. Cambridge, Mass: Addison-Wesley. 